\title{
An optimization technique for speaker mapping neural networks using minimal classification error criterion
}

\author{
Kentaro Kurinami and Masahide Sugiyama \\ ATR Interpreting Telephony Research Laboratories, \\ 2-2 Hikaridai, Seika-cho, Soraku-gun, Kyoto, 619-02 Japan
}

(Received 21 February 1992)

\begin{abstract}
This paper proposes a new optimization technique for speaker mapping neutral network training using the minimal classification error criterion. Recently, neural network modeling has been widely applied to various fields of speech processing. Most neural network applications are classification tasks; however, one of the authors of this paper proposed a speaker mapping neural network as a non-linear continuous mapping application, and showed its effectiveness. On the other hand, the minimal classification error optimization technique has been proposed and applied to several recognition architectures. Since the conventional speaker mapping neural networks have been trained under the minimal distortion criteria, the minimal classification error optimization technique is expected to provide better speaker mapping neural networks. This paper describes the speaker mapping neural network, the minimal classification error optimization technique, derives the algorithm of the minimal classification error optimization technique in the speaker mapping neural network and investigates the relationship between the derived algorithm and the conventional Back Propagation algorithm. Vowel classification experiments are carried out, showing the effectiveness of the proposed algorithm.
\end{abstract}

Keywords: Minimal classification error training, Neural network, Speaker adaptation, Back Propagation algorithm, Non-linear optimization

PACS number: $43.72 . \mathrm{Ne}$

\section{INTRODUCTION}

This paper proposes a new optimization technique for speaker mapping neural network training using the minimal classification error ${\text { criterion. }{ }^{1)}}$ Recently, neural network modeling has been widely applied to various fields of speech processing. Most neural network applications are classification tasks ${ }^{2)}$; however, one of the authors of this paper has proposed a speaker mapping neural network for a continuous mapping task, and showed its effectiveness. ${ }^{3-5)}$ A speaker mapping neural network was originally proposed in frame based mapping mode. ${ }^{6)}$ This speaker mapping technique was extended to segment based mapping and combined with a TDNN-LR continuous speech recognizer. ${ }^{7,8)}$ The conventional speaker mapping neural networks were trained under minimal distortion criteria, which indicate the closeness of the output vectors to the desired vectors.

In addition to minimal distortion criterion, the minimal classification error optimization technique has recently been proposed for several recognition tasks. $^{9-11)}$ This technique was implemented in the continuous density HMM speech recognizer and improves recognition performance. ${ }^{12-14)}$

Most applications to date have been directed towards the improvement of recognition, but the minimal classification error offers a new possibility for training a speaker mapping neural network. While the minimal distortion criterion requires correspondence between input and target vectors, 
the minimal classification error criterion does not require any such correspondence. When a speaker mapping neural network is applied to speech recognizers, it is important that each class (e.g. phoneme) of the input speaker should be transformed to the same class of the standard speaker. Thus minimizing the classification error in speaker mapping provides a better correspondence between two speakers.

In Ref. 15), a neural network feature extractor (TDNN phoneme scanner) was combined with an HMM speech recognizer and its effectiveness was reported in phoneme recognition experiments. However, in this case, the TDNN part and the HMM part were independently trained, thus the TDNN-HMM recognizer was not totally optimized. In this paper, the minimal classification error optimization technique is applied to train the speaker mapping neural network. This paper suggests a new way of combining a neural network based feature extractor with existing speech recognizers.

This paper contains the following sections: in the second section, the speaker mapping neural network is described. In the third section, the minimal classification error optimization technique is described. In the fourth section, the minimal classification optimization technique in the speaker mapping neural network is formulated and a new Back Propagation algorithm is introduced. In the fifth section, vowel classification experiments are carried out, showing the effectiveness of the proposed algorithm.

\section{SPEAKER MAPPING NEURAL NETWORK}

Let $U=\left\{u_{i}\right\}$ be a set of vectors from a standard speaker and $V=\left\{v_{j}\right\}$ be a set of vectors from an unknown input speaker and $f$ be a mapping from $V$ to $U$.

$$
f: V \rightarrow U .
$$

The mapping $f$ is made so that each element in the set $V$ is transformed into a vector of the same category in the set $U$. Speaker mapping is done by transforming the input speaker vector into the standard speaker vector according to $f$. Figure 1 shows the concept of a speaker mapping neural network. To reduce the amount of calculation, speech data of a standard speaker are vector-quantized and some representative vectors are determined in every category.

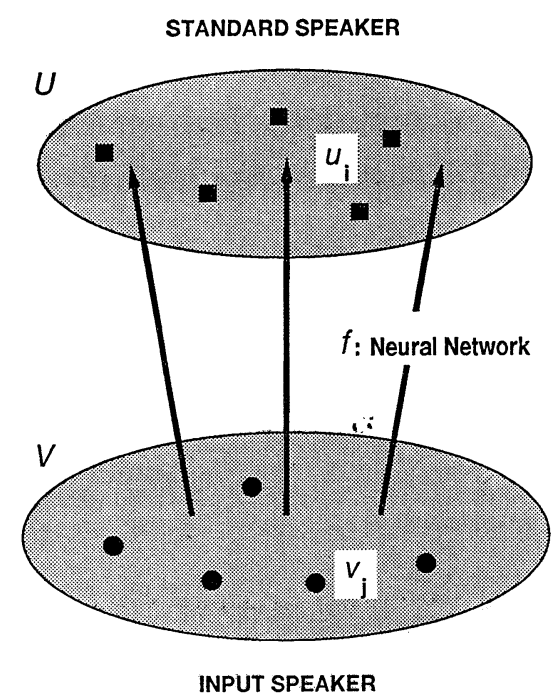

Fig. 1 Concept of speaker mapping.

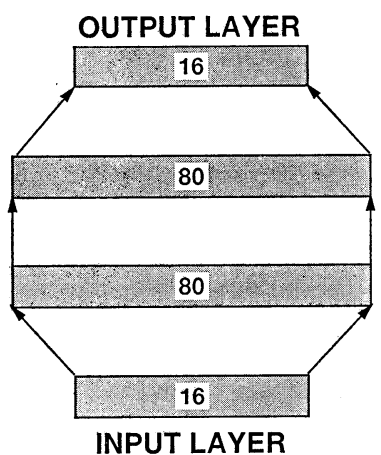

Fig. 2 Speaker mapping neural network.

The speaker mapping neural network is the neural network that performs the mapping from an unknown input speaker vector to a standard speaker vector. It is a four-layered feed-forward neural network as shown in Fig. 2. The number of units is set to 16 in the input and output layers, and 80 in the first and second hidden layers. The number of units in the hidden layers is set larger than the number of input and output layers to achieve sufficient mapping performance. The sigmoid function is used as a non-linear activation function in the hidden layers.

To get the mapping from $V$ to $U$, the network is trained so that one of the standard speaker's vector $u$, which belongs to the same category as $v$, is the closest to $f(v)$. Here, $v$ is an input speaker vector, 


\section{K. KURINAMI and M. SUGIYAMA: AN OPTIMIZATION TECHNIQUE FOR SPEAKER MAPPING}

$f(v)$ is an output vector of the neural network and $u$ is a standard speaker vector. In a conventional Back-Propagation learning algorithm, ${ }^{16)}$ the distance between $f(v)$ and a desired $u$ is regarded as an error function, and the network is trained so that it becomes minimum as follows:

$$
\min _{j} \sum_{v \in V}\|f(v)-u\|^{2}
$$

In this case any relation with other categories is ignored. However, it is more important that an input vector be mapped closer to a vector of the correct category than to that of the wrong one. In this paper, to achieve the object misclassification is regarded as an error function and the network is trained so that it becomes minimum.

\section{MINIMAL CLASSIFICATION ERROR OPTIMIZATION TECHNIQUE}

When each training pattern belongs to either of $K$ categories, optimization using the minimal error criterion trains a recognition system so that the error discrimination rate becomes minimum.

For optimization, the following four functions must be defined.

1. Discriminant function

2. Misclassification measure

3. Loss function

4. Total loss function

\subsection{Discriminant Function}

The discriminant function, $g_{k}(v, \Lambda)$, indicates the degree to which an input vector $v$ belongs to the $k$-th category under $\Lambda$. Here, $\Lambda$ is a set of a system parameters. The category for which the discriminant function is minimum (maximum) is chosen as the correct category. While we discuss only the case where the discriminant function is minimum, there is no lack of generality.

\subsection{Misclassification Measure}

The misclassification measure, $d_{\alpha}(v, \Lambda)$, indicates the extent to which an input vector $v$ is classified into the correct category as compared with other categories, generally defined using discriminant function as follows:

$$
d_{\alpha}(v, \Lambda)=g_{\alpha}(v, \Lambda)-\left[\frac{1}{K-1} \sum_{k, k \neq \alpha} g_{k}(v, \Lambda)^{5}\right]^{1 / \xi} \text {. }
$$

Here, $\alpha$ is the category to which an input vector $v$ belongs and $\zeta$ is a positive real number. By changing $\zeta$, the influence of each category can be con- trolled. In the case of $\zeta \rightarrow \infty, d_{\alpha}(v, A)$ converges as follows:

$$
d_{\alpha}(v, \Lambda)=g_{\alpha}(v, \Lambda)-g_{\beta}(v, \Lambda) .
$$

Here, $\beta$ is the category in which the misclassification measure, except $\alpha$, is minimum. When the misclassification measure is negative, the input vector is classified correctly, and when it is positive, the input vector is classified erroneously.

\subsection{Loss Function}

The loss function, $l_{\alpha}(v, \Lambda)$, indicates if an input vector is correctly classified. $l_{\alpha}(v, A)$ is ideally described as

$$
l_{\alpha}(v, \Lambda)= \begin{cases}0 & \left(d_{\alpha}(v, \Lambda) \leq 0\right) \\ 1 & \left(d_{\alpha}(v, \Lambda)>0\right) .\end{cases}
$$

When an input vector is correctly classified, the loss function is 0 , and when an input vector is erroneously classified, it is 1 . It is difficult to optimize this in Eq. (5), so non linear differential functions are practically applied.

\subsection{Total Loss Function}

$L(\Lambda)$ is the total for the loss function of all input vectors and calculated as:

$$
L(\Lambda)=\sum_{j} l_{\alpha}\left(v_{j}, \Lambda\right) .
$$

$L(\Lambda)$ corresponds to the sum of the misclassifications, so if we regard $L(\Lambda)$ as an objective function and minimize it, we can minimize misclassification.

The conventional gradient descent method can be applied to minimize $L(\Lambda)$. When $\Lambda$ is regarded as a function of time $t$, the change in $L(\Lambda)$ by iteration is calculated as:

$$
\Lambda(t+1)=\Lambda(t)+\delta \Lambda(t),
$$

where, $\delta \Lambda(t)$ is the amount by which $\Lambda$ changes and is given by

$$
\delta \Lambda(t)=-\epsilon(t) \nabla L(\Lambda) .
$$

$\epsilon(t)$ is a step width of parameter estimation, and a positive real number and a variable which controls the degree to which $\Lambda$ changes. In this way, we can achieve optimization and reduce misclassification to a minimum.

\section{MCE OPTIMIZATION APPLIED TO SPEAKER MAPPING NEURAL NETWORK}

\subsection{Formulation}

In the previous section, we explained the general 
form of optimization using minimal classification error (MCE) criterion. In this section, it is combined to a speaker mapping neural network and give its formula.

To maintain generality, we describe an $m$-layered feed-forward neural network with linear activation function units in the input and output layers, and non-linear activation function (sigmoid function) units in the hidden layers. Assume that $i_{y}{ }^{n}$ is the input value to the $y$-th unit in the $n$-th layer, $o_{y}{ }^{n}$ is the output from the $y$-th unit in the $n$-th layer, and $w_{x}{ }^{n-1}{ }^{n}{ }^{n}$ is the connection weight from the $x$-th unit in the $(n-1)$-th layer to the $y$-th unit in the $n$-th layer. The outputs from the units in the $m$-th layer are the network output. Figure 3 shows the relationship between the input and output at the $x$-th unit in the $n$-th layer.

$$
\begin{aligned}
& o_{x}{ }^{n}=\sigma\left(i_{x}{ }^{n}\right) \quad(n=1, \ldots, m), \\
& i_{x}{ }^{n}=\sum_{x^{\prime}} w_{x^{\prime}}{ }^{n-1}{ }_{x}^{n} o_{x^{\prime}}{ }^{n-1} .
\end{aligned}
$$

When $n=m, \sigma$ is an identical mapping in Eq. (9). The system parameter vector, $A$, is equal to the connection weights $\left(w_{x}^{n-1}{ }_{y}^{n}\right)$ of the network, and the outputs of the network depend on inputs and $A$.

Let $U$ and $V$ have the following disjoint partitions;

$$
\begin{array}{lll}
U=U_{1} \cup U_{2} \cup \ldots \cup U_{K}, & U_{i} \cap U_{j}=\phi(i \neq j), \\
V=V_{1} \cup V_{2} \cup \ldots \cup V_{K}, & V_{i} \cap V_{j}=\phi(i \neq j) .
\end{array}
$$

The subsets, $U_{k}$ and $V_{k}$, are corresponding to $k$-th categories in $U$ and $V$, respectively. Suppose that $u_{i}{ }^{k}$ is the $i$-th standard vector which belongs to the $k$-th category $\left(u_{i}{ }^{k}\right.$ : the $y$-th component of $\left.u_{i}{ }^{k}\right), v_{j}$ is the $j$-th vector of the input speaker, and $f\left(v_{j}\right)$ is the output vector of the network. The discriminant function is represented using the Euclidean distance between the output vector and the standard speaker vector.

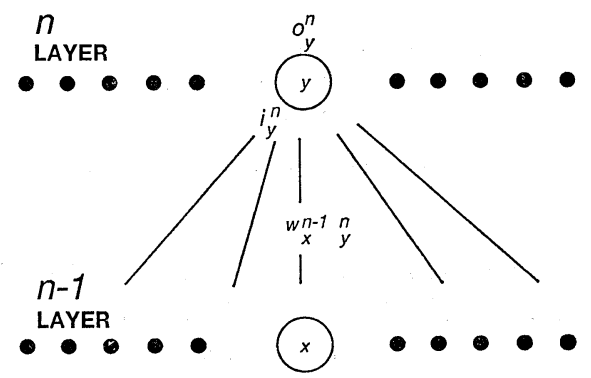

Fig. 3 Definition of connection weights in neural network.

$$
g_{k}(v, \Lambda)=\left\{\sum_{i}\left(|| f(v)-u_{\imath}^{k}||^{2}\right)^{-\eta}\right\}^{-1 / \eta} .
$$

When $\eta \rightarrow \infty$, Eq. (12) converges as follows:

$$
g_{k}(v, \Lambda)=\min _{i}|| f(v)-u_{i}^{k} \|^{2} .
$$

In this paper the simplified equation Eq. (13) is used as a misclassification measure. The sigmoid function is applied to calculate the loss $l_{\alpha}(v, \Lambda)$.

$$
l_{\alpha}(v, \Lambda)=\sigma\left(d_{\alpha}\right)=\frac{1}{1+e^{-d_{\alpha \alpha}}} .
$$

Equation (6) is used as the total of the loss function.

To calculate the degree of change of vector $\Lambda$, partial derivatives of the objective function $L(\Lambda)$ by the connection weights are calculated. $\alpha$ and $\beta$ are the same as in the previous section. $i_{1}$ and $i_{2}$ are defined as follows:

$$
\begin{aligned}
& i_{1}=\arg \min _{i}\left\|f\left(v_{j}\right)-u_{i}{ }^{\alpha}\right\|^{2}, \\
& i_{2}=\arg \min _{i}\left\|f\left(v_{j}\right)-u_{i}{ }^{\beta}\right\|^{2} .
\end{aligned}
$$

\subsection{Calculation of $\nabla L(\Lambda)$}

First, the partial derivatives in the output layer are calculated.

$$
\begin{aligned}
& \frac{\partial L}{\partial w_{x}^{m-1}{ }_{y}^{m}}=\frac{\partial}{\partial w_{x}{ }^{m-1} y^{m}} \sum_{j} l_{\alpha}\left(v_{j}, \Lambda\right) \\
& =\sum_{j} \frac{\partial}{\partial w_{x}{ }^{m-1}{ }_{y}^{m}} \sigma\left(d_{\alpha}\right) \\
& =\sum_{j} \sigma\left(d_{\alpha}\right)\left\{1-\sigma\left(d_{\alpha}\right)\right\} \\
& \times \frac{\partial}{\partial w_{x}{ }^{m-1}{ }_{y}^{m}}\left\{g_{\alpha}\left(v_{j}, \Lambda\right)-g_{\beta}\left(v_{j}, \Lambda\right)\right\} \\
& =\sum_{j} \sigma\left(d_{\alpha}\right)\left\{1-\sigma\left(d_{\alpha}\right)\right\} \\
& \times \frac{\partial}{\partial w_{x}{ }^{m-1}{ }_{y} m}\left\{\left\|f\left(v_{j}\right)-u_{i_{1}}{ }^{\alpha}\right\|^{2}\right. \\
& \left.-\left\|f\left(v_{j}\right)-u_{i_{2}}{ }^{\beta}\right\|^{2}\right\} \\
& =\sum_{j} \sigma\left(d_{\alpha}\right)\left\{1-\sigma\left(d_{\alpha}\right)\right\} \\
& \times\left[\left\{2\left(o_{y}{ }^{m}-u_{i_{1}}{ }^{\alpha}\right) \frac{\partial}{\partial w_{x}{ }^{m-1}{ }_{y}{ }^{m}} o_{y}{ }^{m}\right\}\right. \\
& \left.-\left\{2\left(o_{y}{ }^{m}-u_{i_{2}}{ }^{\beta}\right) \frac{\partial}{\partial w_{x}{ }^{m-1}{ }^{m}} o_{y}{ }^{m}\right\}\right] \\
& =\sum_{j} 2 \sigma\left(d_{\alpha}\right)\left\{1-\sigma\left(d_{\alpha}\right)\right\} \\
& \times\left\{\left(o_{y}{ }^{m}-u_{i_{1}}{ }^{\alpha}\right)-\left(o_{y}{ }^{m}-u_{i_{2}}{ }^{\beta}\right)\right\}
\end{aligned}
$$




\section{K. KURINAMI and M. SUGIYAMA: AN OPTIMIZATION TECHNIQUE FOR SPEAKER MAPPING}

$$
\begin{aligned}
& \times \frac{\partial}{\partial{w_{x}}^{m-1}{ }_{y}^{m}} \sum_{x^{\prime}}{w_{x^{\prime}}}^{m-1}{ }_{y}^{m} \boldsymbol{O}_{x^{\prime}}{ }^{m-1} \\
& =\sum_{j} 2 \sigma\left(d_{\alpha}\right)\left\{1-\sigma\left(d_{\alpha}\right)\right\} \\
& \times\left(u_{i_{2}}{ }^{\beta}{ }_{y}-u_{i_{1}}{ }^{\alpha}\right){ }_{x}{ }^{m-1}
\end{aligned}
$$

Here, $\delta_{m y}$ is the error magnitude.

$$
\delta_{m y}=2 \sigma\left(d_{\alpha}\right)\left\{1-\sigma\left(d_{\alpha}\right)\right\}\left(u_{i_{2}{ }^{\beta}}{ }_{y}-u_{i_{1}}{ }^{\alpha}\right) .
$$

The Eq. (17) is transformed as follows:

$$
\frac{\partial L}{\partial w_{x}{ }^{m-1}{ }^{m}}=\sum_{j} \delta_{m y} o_{x}{ }^{m-1} \text {. }
$$

Next, the partial derivatives in the $n$-th layer are calculated.

$$
\begin{aligned}
& \frac{\partial L}{\partial w_{x}^{n-1} y^{n}}=\frac{\partial}{\partial w_{x}^{n-1} y^{n}} \sum_{j} l_{\alpha}\left(v_{j}, A\right) \\
& =\sum_{j} \sigma\left(d_{\alpha}\right)\left\{1-\sigma\left(d_{\alpha}\right)\right\} \\
& \times \sum_{z}\left[\left\{2\left(o_{z}{ }^{n+1}-u_{i_{1}}{ }^{\alpha}\right)\right.\right. \\
& \left.\left.-2\left(o_{z}{ }^{n+1}-u_{i_{2}}{ }^{\beta}\right)\right\} \frac{\partial}{\partial w_{x}^{n-1} y^{n}} o_{z}^{n+1}\right] \\
& =\sum_{j} \sum_{z} \delta_{(n+1) z} \frac{\partial}{\partial w_{x}^{n-1}{ }_{y}^{n}} w_{y z}^{n{ }^{n+1}} o_{y}{ }^{n} \\
& =\sum_{j} \sum_{z} \delta_{(n+1) z} w_{y z}^{n{ }^{n+1}} \\
& \times \frac{\partial}{\partial w_{x}^{n-1} y^{n}} \sigma\left(\sum_{x^{\prime}}{w_{x^{\prime}}}^{n-1} y^{n} o_{x^{\prime}}{ }^{n-1}\right) \\
& =\sum_{j} \sum_{z} \delta_{(n+1) z} w_{y}^{n}{ }^{n+1} o_{y}{ }^{n}\left(1-o_{y}{ }^{n}\right) o_{x}{ }^{n-1}
\end{aligned}
$$

The error magnitude $\delta_{n y}$ is defined as

$$
\delta_{n y}=\sum_{z} \delta_{(n+1) z} w_{y z}{ }^{n+1} o_{y}{ }^{n}\left(1-o_{y}{ }^{n}\right) .
$$

The Eq. (20) is transformed as follows:

$$
\frac{\partial L}{\partial w_{x}^{n-1}{ }_{y}^{n}}=\sum_{j} \delta_{n y} o_{x}^{n-1} .
$$

The learning rule of the connection weights is determined by Eqs. (7), (8), (19) and (22) as follows:

$$
w_{x}^{n-1} y^{n}(t+1)=w_{x}^{n-1}{ }_{y}^{n}(t)-\epsilon(t) \sum_{j} \delta_{n y} o_{x}{ }^{n-1} .
$$

\subsection{Meaning of Derived Algorithm}

Here, we consider the meaning of the derived learning rule as compared with the conventional Back-Propagation. Equations (19) and (22) have the same formulae as those of the conventional BackPropagation, and both the $\delta_{n y}$ of the proposed method and that of Back-Propagation are calculated using $\delta_{(n+1) z}$. This indicates that the learning algorithm is equal to the conventional Back-Propagation in propagating the error backward from the output side to the input side, but they have different objective functions and their propagating errors are completely different. In the conventional BackPropagation the difference between the actual output and the desired output, $\left(o_{y}{ }^{m}-u_{i_{1}}{ }^{\alpha}\right)$, is used as the error. On the other hand, the error of the proposed learning rule has two remarkably different points. The first point is $\left(\sigma\left(d_{\alpha}\right)\left(1-\sigma\left(d_{\alpha}\right)\right)\right)$. It is derived by differentiating the loss function, which is defined using the sigmoid function. This value has the following inequality:

$$
\begin{gathered}
-\infty<d_{\alpha}<+\infty, \\
0<\sigma\left(d_{\alpha}\right)\left(1-\sigma\left(d_{\alpha}\right)\right) \leq \frac{1}{4} .
\end{gathered}
$$

The closer $d_{\alpha}$ is to 0 , the larger it becomes and thus the degree to which the parameter changes is also large. When $d_{\alpha}$ approaches 0 , the difference between the output vector and the desired vector is almost equal to the difference between the output vector and the undesired vector, that is to say, the output vector is near the category boundary. In conclusion, by introducing the loss function, the derived learning rule has a greater effect on the category boundary where misclassification occurs. The second point is $\left(u_{i_{2}{ }^{\beta} y}{ }^{\beta}-u_{i_{1}}{ }^{\alpha}\right)$. It shows the difference between the desired standard speaker vector and the nearest wrong standard speaker vector. The greater the distance between the wrong standard speaker vector and the desired one, the more the parameter changes. Using this as the error means that proposed algorithm takes account of the relation between categories.

\section{VOWEL CLASSIFICATION EXPERIMENTS}

The optimized speaker mapping neural network using the minimal classification error technique is evaluated using 5 Japanese vowel classification experiments. ${ }^{17}{ }^{18)}$ Experimental conditions are shown in Table 1. Each sample was extracted from the middle of the vowel.

\subsection{Step Width of Parameter Estimation $(\epsilon(t))$}

In the preliminary experiments the step width of parameter estimation, $\epsilon(t)$, was set to a monotonic increasing function, $\epsilon(t)=1 / t$, where $t$ is the number 
Table 1 Experimental conditions.

\begin{tabular}{|c|c|c|}
\hline \multicolumn{2}{|c|}{ Speaker } & $\begin{array}{l}\text { Standard speaker: Male (M1) } \\
\text { Unknown speaker: Male (M2) }\end{array}$ \\
\hline \multicolumn{2}{|c|}{ Task } & 5 Vowel $\mid a, i, u, e, o /$ \\
\hline \multicolumn{2}{|c|}{ Feature parameter } & 16th LPC cepstrum \\
\hline \multirow{4}{*}{$\begin{array}{l}\text { Analysis } \\
\text { condi- } \\
\text { tion }\end{array}$} & $\begin{array}{l}\text { LPC analy- } \\
\text { sis order }\end{array}$ & 14th order \\
\hline & $\begin{array}{l}\text { Sampling } \\
\text { frequency }\end{array}$ & $12 \mathrm{kHz}$ \\
\hline & $\begin{array}{l}\text { Window } \\
\text { length }\end{array}$ & 256 points $(21.3 \mathrm{~ms})$ \\
\hline & $\begin{array}{l}\text { Window } \\
\text { function }\end{array}$ & Hamming \\
\hline \multicolumn{2}{|c|}{$\begin{array}{l}\text { VQ codebook } \\
\text { size }\end{array}$} & $\begin{array}{l}\quad 40(8 \times 5 \text { vowels }) \\
\text { category dependent generation }\end{array}$ \\
\hline \multirow{3}{*}{$\begin{array}{l}\text { Speech } \\
\text { data }\end{array}$} & $\begin{array}{l}\text { Codebook } \\
\text { gener- } \\
\text { ation }\end{array}$ & $\begin{array}{l}500 \text { samples }(100 \times 5 \text { vowels }) \\
\text { (Male M1 5,240 words-even } \\
\text { numbered) }\end{array}$ \\
\hline & $\begin{array}{l}\text { Adapta- } \\
\text { tion } \\
\text { sample }\end{array}$ & $\begin{array}{l}500 \text { samples }(100 \times 5 \text { vowels }) \\
\text { (Male M2 5,240 words-even } \\
\text { numbered) }\end{array}$ \\
\hline & $\begin{array}{l}\text { Test } \\
\text { sample }\end{array}$ & $\begin{array}{l}500 \text { samples }(100 \times 5 \text { vowels }) \\
\text { (Male M2 5,240 words-odd } \\
\text { numbered) }\end{array}$ \\
\hline
\end{tabular}

of learning iterations. In that case, however, the output values differed considerably from the desired ones after several learning iterations. When the output values differ considerably from the desired values, learning will no longer be carried out, even if misclassifications occur. Any more improvement in learning can not be expected. Since $\epsilon(t)$ is too large in the beginning of the learning stage, $\epsilon(t)$ is modified using the following equation:

$$
\epsilon(t)=\frac{1}{t+a} .
$$

In this way, divergence of learning could be prevented. When $a$ was more than 10 , the parameter vector was optimized stably. When $a$ was too large, it took many iterations to converge. Thus, $a$ was set to 10 in the following experiments.

\subsection{Choice of Loss Functions}

In the previous section the sigmoid function (Eq. (14)) was applied as the loss function; however, the loss function has the following possibilities:

$$
l_{\alpha}(v, \Lambda)=\left\{\begin{array}{ll}
0 & \left(d_{\alpha}(v, \Lambda) \leq 0\right) \\
\left(d_{\alpha}\right)^{\xi} & \left(d_{\alpha}(v, \Lambda)>0\right)
\end{array} \quad(0<\xi \ll 1)\right.
$$

Figure 4 shows the difference in the characteristics of the above loss functions. In the case of using Eq. (26), however, though there was some improvement in classification accuracy, the problem was that classification accuracy was not stabilized. The reason may be that it is difficult to chose an appropriate value for $\xi$. The number of misclassifications through learning is shown in Fig. 5, Fig. 6 and Fig. 7. Figure 5 shows the case of $\xi=0.01$ and Fig. 6 shows the case of $\xi=0.001$. In Fig. 5, the number of misclassifications fluctuates considerably. In Fig. 6, though it is better than in Fig. 5, it still fluctuates. Lowering $\xi$ had no further effect. Figure 7 shows the case in which the sigmoid function was used as the loss. There is a small fluctua-

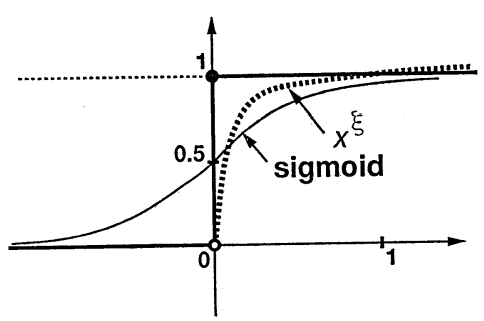

Fig. 4 Characteristics of several loss functions.

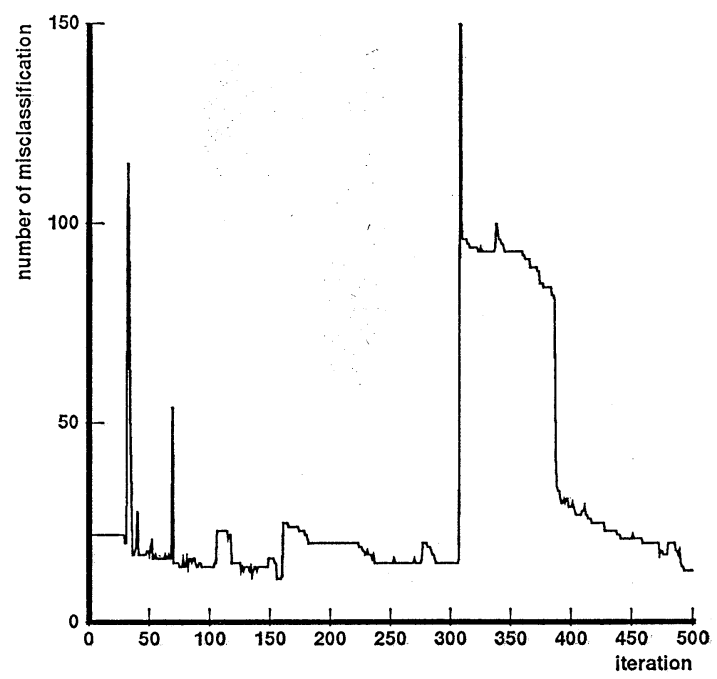

Fig. 5 Converging training procedure (power function $x^{\xi}, \xi=0.01$ ). 


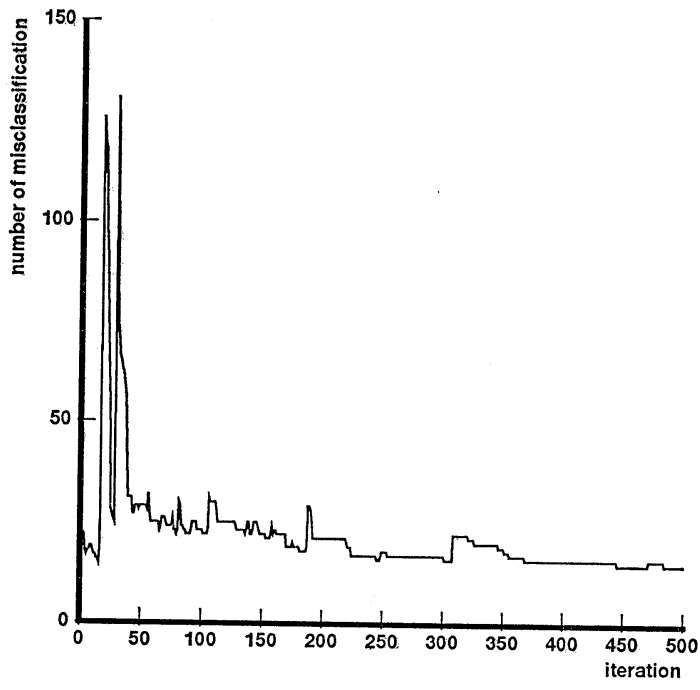

Fig. 6 Converging training procedure (power function $\left.x^{\xi}, \xi=0.001\right)$.

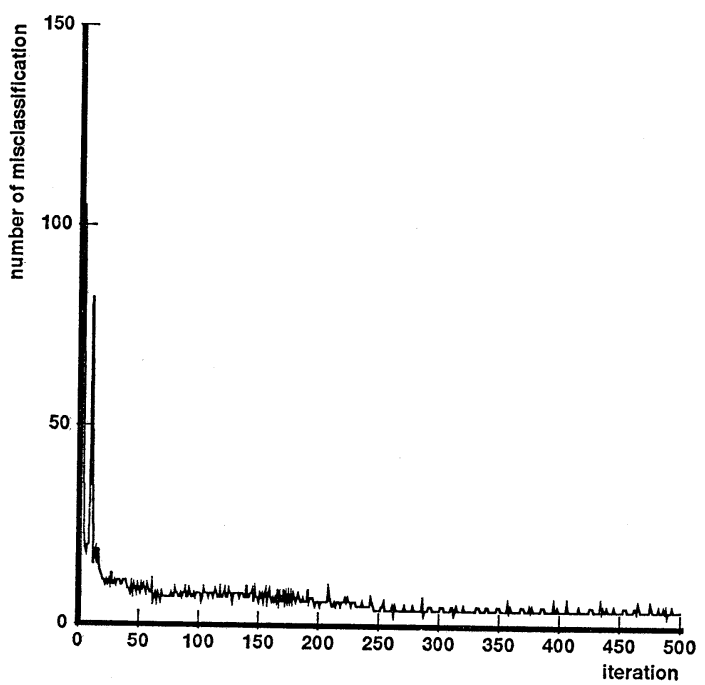

Fig. 7 Converging training procedure (sigmoid function).

tion but, as a whole, it converges well and classification accuracy is good. For this reason, the sigmoid function was used as the loss in subsequent experiments.

\subsection{Initial Connection Weights $(\Lambda)$}

Experiments were performed using two different initial vectors: random values and values obtained
Table 2 Results of vowel classification experiments.

\begin{tabular}{lccc}
\hline Data & Link weights $\Lambda$ & $\begin{array}{c}\text { No opti- } \\
\text { mization }\end{array}$ & $\begin{array}{c}\text { With opti- } \\
\text { mization }\end{array}$ \\
\hline \multirow{2}{*}{$\begin{array}{c}\text { Training } \\
\text { data }\end{array}$} & Random & 20.0 & 99.6 \\
\cline { 2 - 4 } & Unsupervised training & 95.6 & 99.2 \\
\hline \multirow{2}{*}{$\begin{array}{c}\text { Test } \\
\text { data }\end{array}$} & Random & 20.0 & 97.4 \\
\cline { 2 - 4 } & Unsupervised training & 94.4 & 97.2 \\
\hline Classification rate: $\%$ & &
\end{tabular}

from unsupervised training. ${ }^{17,18)}$ The reason that the values from unsupervised learning are used is that these values, which are correct to some degree, seem to converge better.

\subsection{Experimental Results}

The results of the experiments are shown in Table 2 , which shows the classification accuracy for training data and for test data. The classification accuracy is calculated by

$$
R=\frac{N_{s}}{N_{a}} \times 100,
$$

$N_{a}$ shows the number of all samples and $N_{s}$ shows the number of samples whose $g_{\alpha}$ is smaller than $g_{\beta}$.

The result shows the classification accuracy is $99.6 \%$ for training data and $97.4 \%$ for test data by optimizing the speaker mapping neural network using the minimal classification error criterion. The difference between the initial connection weights had almost no influence. Compared with unsupervised learning, the proposed learning method improved classification accuracy. Since the supervised learning $^{3,4,6)}$ using an conventional DTW with 25 words resulted in a classification accuracy of $95.8 \%$, and the tasks were different and could not be compared directly, the proposed learning method achieved a higher classification accuracy.

\section{CONCLUSIONS}

An optimization technique using the minimal classification error criterion was applied to training a speaker mapping neural network, and its formulation was described. Its main features are that the difference between the desired outputs and the wrong outputs is used as the error, and that the 
category boundary tends to be well trained. Experiments on speaker mapping with 5 vowels was performed, and achieved a classification accuracy of $99.6 \%$ for training data and $97.4 \%$ for test data. When a neural network is regarded as a feature extractor, one solution to the optimal design problem of a feature extractor was given.

Finally, we suggest further studies.

- comparing the classification accuracy of the technique with the general value ( $\zeta$ in Eq. (3) and $\eta$ in Eq. (12)).

- applying the technique to other phoneme categories.

- extending the technique to a speaker mapping neural network in DTW or HMM systems.

- extending the technique to a general neural network.

\section{ACKNOWLEDGEMENTS}

The authors would like to thank Prof. M. Nakagawa and Dr. A. Kurematsu for their support in this research, Mr. K. Fukuzawa for information about neural network software tools, and Dr. S. Katagiri, ATR Auditory \& Visual Perception Labs., for his valuable comments and suggestions.

\section{REFERENCES}

1) M. Sugiyama and K. Kurinami, "An optimization of speaker mapping neural network using minimal classification error criterion," Proc. Autumn Meet. Acoust. Soc. Jpn. 2-Q-22, 201-202 (1992) (in Japanese).

2) A. Waibel, T. Hanazawa, G. Hinton, K. Shikano, and $\mathrm{K}$. J. Lang, "Phoneme recognition using timedelay neural networks," IEEE Trans. Acoust. Speech Signal Process. ASSP-37, 328-339 (1989).

3) K. Fukuzawa, H. Sawai, and M. Sugiyama, "Speaker adaptation using identity mapping by neural networks," Proc. Autumn Meet. Acoust. Soc. Jpn. 1-8-16, 31-32 (1990) (in Japanese).

4) K. Fukuzawa, H. Sawai, and M. Sugiyama, "Segment-based speaker adaptation by neural network," Proc. IEEE-SP Workshop on Neural Networks for Signal Processing, 440-451 (Oct. 1991).

5) K. Fukuzawa, Y. Komori, H. Sawai, and M. Sugiyama, "A segment-based speaker adaptation neural network applied to continuous speech recognition," Proc. Autumn Meet. Acoust. Soc. Jpn. 3-5-10, 109110 (1991) (in Japanese).

6) K. Iso, M. Asogawa, K. Yoshida, and T. Watanabe, "Speaker adaptation using neural network," Proc. Spring Meet. Acoust. Soc. Jpn. 1-6-16, 31-32 (1989) (in Japanese).
7) Y. Minami, M. Miyatake, H. Sawai, and K. Shikano, "Continuous speech recognition using TDNN phoneme spotting and generalized LR parser," Proc. Autumn. Meet. Acoust. Soc. Jpn. 3-1-11, 97-98 (1989) (in Japanese).

8) K. Fukuzawa, Y. Komori, H. Sawai, and M. Sugiyama, "A segment-based speaker adaptation neural network applied to continuous speech recognition," Proc. ICASSP-92, 55.1 (1992).

9) S. Katagiri, C. H. Lee, and B. H. Juang, "A generalized probabilistic descent method," Proc. Autumn. Meet. Acoust. Soc. Jpn. 2-P-6, 141-142 (1990).

10) E. MacDermott and S. Katagiri, "Discriminative training for various speech units," Tech. Rep. Acoust. Soc. Jpn. SP91-12, 47-54 (1991).

11) S. Katagiri, C. H. Lee, and B. H. Juang, "Discriminative multi-layer feed-forward networks," Proc. IEEE-SP Workshop Neural Networks for Signal Processing, 11-20 (1991).

12) D. Rainton and S. Sagayama, "Minimum error classification training of HMMs-Implementation details and experimental results-," Tech. Rep. Acoust. Soc. Jpn. SP91-107, 39-46 (1992).

13) A. Duchon, D. Rainton, and S. Katagiri, "Implementing the generalized probabilistic descent method on continuous HMMs," Proc. Spring Meet. Acoust. Soc. Jpn. 1-1-1, 1-2 (1992).

14) D. Rainton and S. Sagayama, "Reducing errors using minimum error training of continuous mixture density HMMs," Proc. Spring Meet. Acoust. Soc. Jpn. 1-1-2, 3-4 (1992).

15) M. Sutiyama and A. Biem, "TDNN-HMM approach to phoneme recognition," Proc. Autumn Meet. Acoust. Soc. Jpn. 2-P-6, 149-150 (1991) (in Japanese).

16) D. E. Rumelhart, G. E. Hinton, and R. J. Williams, Parallel Distributed Processing: Explorations in the Microstructure of Cognition, Vol. 1: Foundations (MIT Press, Cambridge MA, 1986).

17) M. Sugiyama, K. Fukuzawa, H. Sawai, and S. Sagayama, "Unsupervised training methods for set mappings using neural networks," Proc. Autumn Meet. Acoust. Soc. Jpn. 2-P-10, 149-150 (1990) (in Japanese).

18) K. Fukuzawa and M. Sugiyama, "Evaluation of unsupervised speaker adaptation using a neural network," Proc. Autumn Meet. Acoust. Soc. Jpn. 3-5-11, 111-112 (1991) (in Japanese).

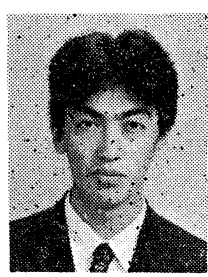

Kentaro Kurinami was born in Tokyo, Japan in 1969 . He graduated from the Department of Electronics, Keio University in 1990 . He is currently studying for the master degree at Keio University. 


\section{K. KURINAMI and M. SUGIYAMA: AN OPTIMIZATION TECHNIQUE FOR SPEAKER MAPPING}

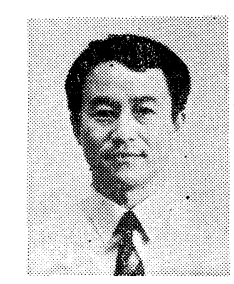

Masahide Sugiyama

was born in Tochigi, Japan in 1954. After receiving the master degree of science from Tohoku University in 1979, he joined NTT Musashino Electric Communication Laboratories (NTT Musashino Research Center). He received the $\mathrm{Ph}$.

$\mathrm{D}$ degree in engineering from Tohoku University in 1985. He was a visiting researcher at AT \& T Bell Laboratories from Nov. 1986 to Nov. 1987.
Through NTT basic Research Laboratories, he is currently a supervisor at ATR Interpreting Telephony Research Laboratories. His research interests are LPC spectral distance measures, Vector Quantization applied to speech recognition, feature-based speech recognition, unsupervised speaker adaptation, text-independent speaker recognition, speech spectral analysis, information geometric and speech analysis and language recognition using acoustic features. 